\title{
Differential Effects of Inhibitors on Respiratory Activity of Synchronous Cultures of Bacillus subtilis Prepared by Continuous-flow Centrifugation
}

\author{
By C. EDWARDS* AND R. J. MCCANN \\ Department of Microbiology, Life Sciences Building, University of Liverpool, P.O. Box 147, \\ Liverpool L69 3BX, U.K.
}

(Received 9 October 1980; revised 15 December 1980)

\begin{abstract}
Size selection by continuous-flow centrifugation was used to prepare synchronous cultures of Bacillus subtilis. A selection of 5-10\% was required to yield cultures which gave synchrony indices of about $0 \cdot 45$. Respiration rates [ $\mathrm{nmol} \mathrm{O}_{2} \mathrm{~min}^{-1}(\mathrm{ml} \text { culture })^{-1}$ ] of cells growing in the presence of glucose rose discontinuously as two steps in each cell cycle with the mid-points of the rises centred at 0.37 and 0.87 of a cycle. An asynchronous control culture exhibited no such periodicities. Two inhibitors of energy conservation $-N, N^{\prime}$-dicyclohexylcarbodi-imide and 4-chloro-7-nitrobenzofurazan - inhibited respiration maximally at 0.0 and 0.6 of a cycle whilst an inhibitor of respiration - 2-heptyl-4-hydroxyquinoline- $N$-oxide - exerted maximal effect only at 0.4 of a cycle. These results are compared with other temporal events which have been reported to occur during the cell cycle of $B$. subtilis.
\end{abstract}

\section{INTRODUCTION}

Complex changes in respiratory activity occur during the cell cycles of many eukaryotic micro-organisms (Lloyd, 1974). More recently, periodic changes in respiration rates have been reported in synchronous cultures of Alcaligenes eutrophus (Edwards \& Jones, 1977) and Escherichia coli (Evans, 1975). It has been proposed that the pattern of periodicities is influenced by the growth substrate. For example, oxygen uptake rates increase as a series of maxima and minima in $E$. coli growing synchronously in the presence of glucose, whereas in the presence of a non-fermentable carbon source such as glycerol, respiration rates rise in a stepwise fashion (Poole, 1977). A similar effect of the carbon energy source on respiration has been noted for synchronous cultures of the yeast Schizosaccharomyces pombe (Poole \& Lloyd, 1974).

In this paper we report the use of continuous-flow centrifugation (Lloyd et al., 1975) to prepare synchronous cultures of Bacillus subtilis growing in the presence of glucose. Respiration rates of whole cells increased as two steps per cycle. These periodicities were further investigated by the addition of two inhibitors of energy conservation and one of respiration at different stages of the cell cycle. Differential inhibition by all three inhibitors was observed.

\section{METHODS}

Abbreviations. DCCD, $N, N^{\prime}$-dicyclohexylcarbodi-imide; HQNO, 2-heptyl-4-hydroxyquinoline- $N$-oxide; Nbf$\mathrm{Cl}$, 4-chloro-7-nitrobenzofurazan.

Organism and growth conditions. Bacillus subtilis (NC113) was maintained on plates of nutrient agar (Oxoid) and grown on a minimal salts medium supplemented with filter-sterilized glucose $(40 \%, \mathrm{w} / \mathrm{v})$ to a concentration of $50 \mathrm{~mm}$ as described previously (Edwards, 1980).

Preparation of synchronous cultures. Bacteria from the late-exponential phase of growth were used to inoculate three prewarmed 2 litre conical flasks each containing $400 \mathrm{ml}$ of minimal medium to a final $A_{550}$ of $0 \cdot 10-0 \cdot 15$. The cultures were then incubated at $30^{\circ} \mathrm{C}$ in a rotary orbital incubator (Gallenkamp) until the culture density reached 
an $A_{550}$ of $0 \cdot 60-0 \cdot 65$. By this stage the organisms were growing exponentially. The pooled cultures were then passed through a Sharples continuous-flow centrifuge driven by an overhead electric motor (Pennwalt, Camberley, Surrey) at a rotor speed of $16400 \mathrm{rev} . \mathrm{min}^{-1}$ and at a flow rate of approximately $140 \mathrm{ml} \mathrm{min} \mathrm{m}^{-1}$. Under these conditions $5-10 \%$ of the original culture was retained in the rotor effluent. After discarding the first $200 \mathrm{ml}$ of the effluent to allow for equilibration, a portion $(200 \mathrm{ml})$ which contained the smallest size class of bacteria was collected in a prewarmed 2 litre conical flask, immediately returned to the incubator and used as the source of the synchronous culture. The whole procedure, from the removal of the exponentially growing culture to the return of the rotor effluent to the incubator, took approximately $5 \mathrm{~min}$.

Assessment of synchrony. The degree of synchrony in different experiments was expressed by the synchrony index $(F)$ of Blumenthal \& Zahler (1962), as described previously (Edwards, 1980).

Analytical methods. Growth of cultures was monitored by measuring changes in $A_{s 50}$ or by determining cell numbers. For the latter, culture samples $(1 \mathrm{ml})$ were fixed with formaldehyde $(0.4 \%, \mathrm{w} / \mathrm{v})$, diluted in Isoton to approximately $6 \times 10^{5}$ organisms $\mathrm{ml}^{-1}$ and counted using a model ZBI Coulter counter (Coulter Electronics, Harpenden, Herts.) fitted with a $30 \mu \mathrm{m}$ orifice. All counts were corrected for background. Cell volumes were measured using a Channelyser linked to the Coulter counter after calibration with latex beads of $1.15 \mu \mathrm{m}$ diameter.

Oxygen uptake rates were measured polarographically at $30^{\circ} \mathrm{C}$ using a Rank oxygen electrode (Rank Bros., Bottisham, Cambs.) linked to a Vitatron chart recorder (MSE, Crawley, Sussex). Samples (2.4 ml) of undiluted cell suspensions were rapidly transferred from growing cultures into the reaction chamber and oxygen uptake rates were measured for approximately $8 \mathrm{~min}$. Appropriate amounts of each inhibitor were added to the reaction chamber after a linear rate of oxygen uptake had been obtained. $\mathrm{Nbf}-\mathrm{Cl}$ and DCCD were made up as methanolic solutions. Appropriate controls showed that, for the additions employed, methanol had no inhibitory effect on respiration rate. HQNO was made up as an aqueous solution.

\section{RESULTS}

\section{Size selection by continuous-flow centrifugation}

The efficiency of size selection by continuous-flow centrifugation was assessed for $B$. subtilis. Exponentially growing cultures which had an $A_{550}$ between 0.50 and 0.55 were subjected to continuous-flow centrifugation at different rotor speeds. Between $15 \times 10^{3}$ and $21 \times 10^{3}$ rev. $\min ^{-1}, 5-12 \%$ of the cells escaped sedimentation and were recovered in the effluent. Progressively lowering the rotor speed resulted in a sharp increase in the proportion of cells escaping sedimentation. A rotor speed of $16.5 \times 10^{3} \mathrm{rev} . \mathrm{min}^{-1}$ resulted in an effluent containing approximately $10 \%$ of the original population which subsequently grew synchronously with a synchrony index of 0.46 (Fig. 1 c). A rotor speed of $13 \times 10^{3}$ rev. $\min ^{-1}$ resulted in a effluent containing $21 \%$ of the original population and the ensuing growth had only a slight degree of synchrony $(F=0.31$; Fig. $1 b)$. An effluent which contained $60 \%$ (rotor speed $9.0 \times 10^{3} \mathrm{rev} \cdot \mathrm{min}^{-1}$ ) of the original population grew exponentially indicating no size selection or perturbation by the centrifugation procedure (Fig. 1a). For B. subtilis, therefore, a rotor speed of about $16-17 \times 10^{3} \mathrm{rev} \cdot \mathrm{min}^{-1}$ is needed to select a population of synchronously dividing cells. Little perturbation of the cultures following the size-selection procedure was apparent since they all grew with doubling times $(80 \mathrm{~min})$ similar to untreated cells.

\section{Measurements of oxygen uptake rates during synchronous growth}

The number of cells did not increase for $60 \mathrm{~min}$ after size selection by continuous-flow centrifugation (Fig. 2). There was then a synchronous burst of division which lasted approximately $40 \mathrm{~min}$. During this period the number of cells doubled giving a synchrony index of 0.45 . The ensuing lag period lasted only $28 \mathrm{~min}$ and was followed by a second increase in cell numbers which was not as synchronous as the first. The modal cell volume rose to a maximum at 60 and $140 \mathrm{~min}$ and decreased rapidly during the division period indicating that both the step increases in cell numbers were, in fact, synchronous (Fig. 2). The time between the maxima of modal cell volume $(80 \mathrm{~min})$ marked the duration of the cell cycle and was similar to the doubling time of exponentially growing cells. The $A_{550}$ of the culture rose smoothly in an exponential fashion with a doubling time of $80 \mathrm{~min}$ (Fig. 2). In contrast, 


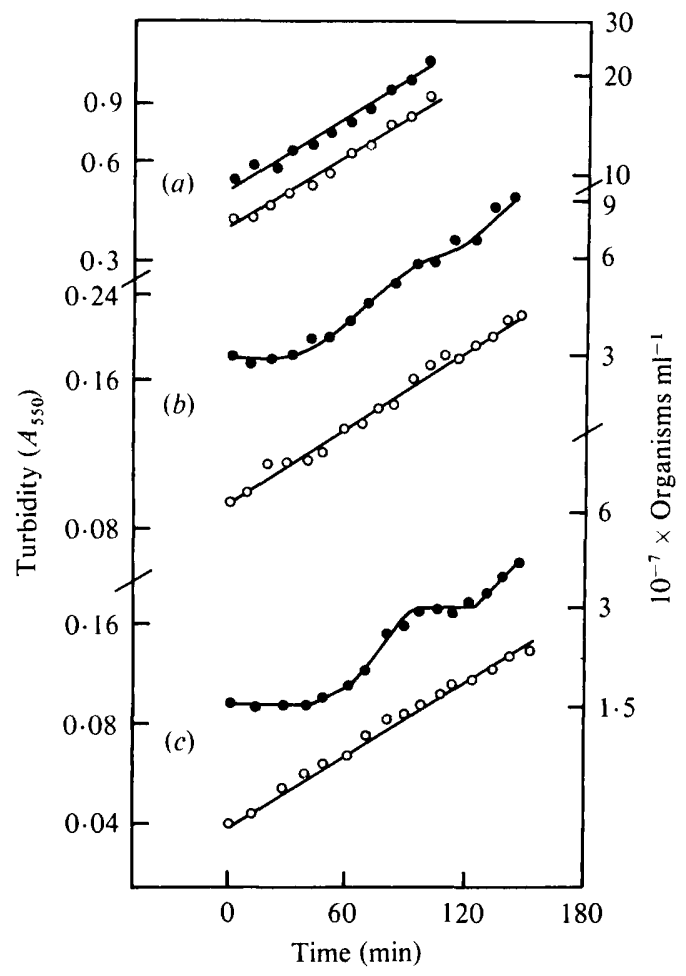

Fig. 1. Efficiency of continuous-flow centrifugation for size selection of $B$. subtilis. Effluents containing $60 \%(a), 21 \%(b)$ and $10 \%(c)$ of the original population were obtained by adjusting the rotor speed. Cell numbers (O) and $A_{550}(O)$ were determined at intervals during subsequent growth.

oxygen uptake rates rose discontinuously as two steps per cycle, but doubled overall in each cell cycle (Fig. 2). The mid-points of the rises in respiration rates were centred at 0.35 and 0.90 of a cell cycle.

In order to assess any effects of the continuous-flow centrifugation procedure on the treated culture, a control experiment was performed. An asynchronous culture was passed through the rotor and the effluent was used to resuspend some of the cells which had sedimented on to the rotor wall thus restoring an asynchronous population of cells. This culture was incubated, and cell numbers, $A_{550}$ and oxygen uptake rates were measured. All three parameters increased in smooth exponential fashion with doubling times of approximately $80 \mathrm{~min}$. No periodicity of respiration rate was apparent after this treatment.

\section{Effects of inhibitors on respiration during synchronous growth}

Inhibitors of oxygen uptake (Slater, 1967) were tested in an attempt to elucidate the basis for the periodic step increases in oxygen uptake rate during synchronous growth. It was found that rotenone and antimycin A (inhibitors of electron transport at sites 1 and 2, respectively) had no effect on respiration. However, HQNO (up to $136 \mathrm{nM}$ ), which inhibits electron transport at site 2 , inhibited oxygen uptake rates of exponentially growing cells by approximately $80 \%$. It was also found that two inhibitors of proton-translocating ATPases, Nbf-Cl (Ferguson et al., 1974) and DCCD (Beechey et al., 1967), inhibited respiration of asynchronous populations of cells. It therefore appeared possible to investigate the contribution of electron transport and energy-transfer reactions to the observed respiratory periodicities.

Addition of $1.66 \mu \mathrm{M}-\mathrm{Nbf}-\mathrm{Cl}$ (sufficient to inhibit the respiration of asynchronously dividing 


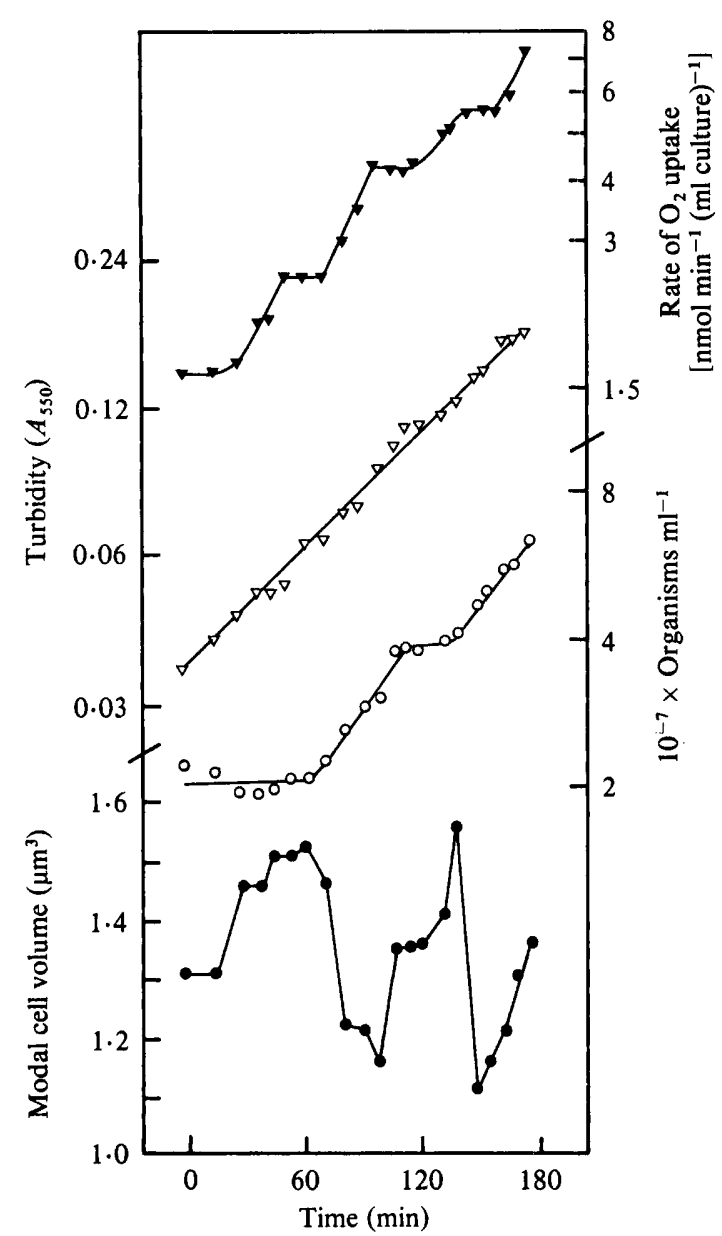

Fig. 2

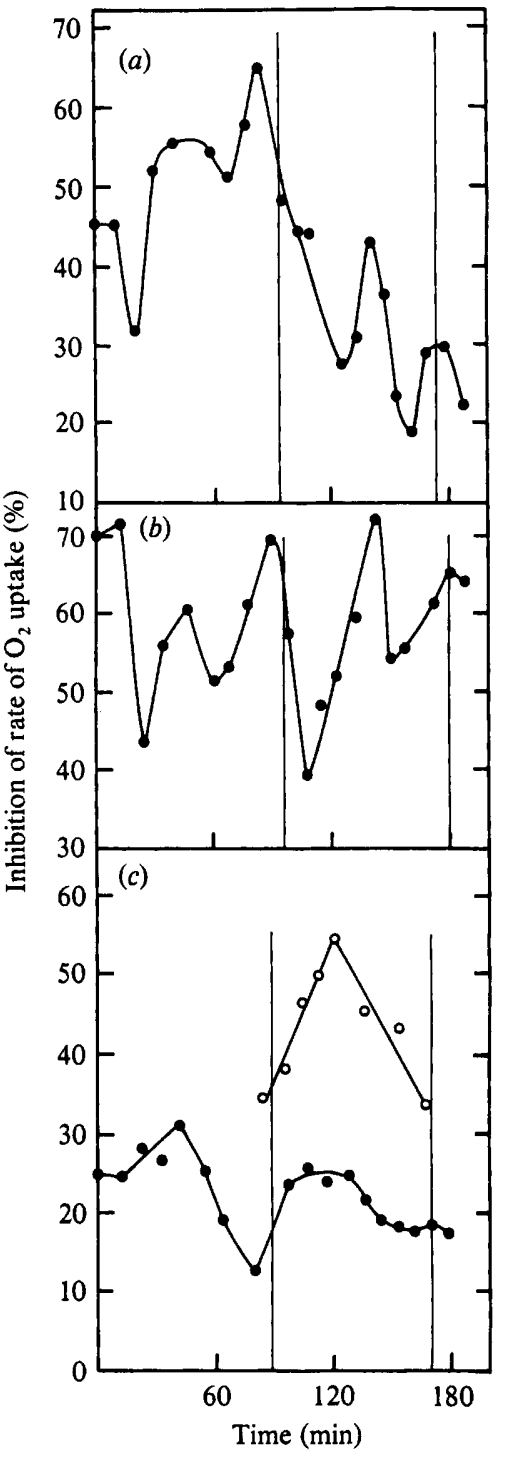

Fig. 3

Fig. 2. Respiration rates at different times during synchronous growth of $B$. subtilis. After size selection by continuous-flow centrifugation at $16.5 \times 10^{3} \mathrm{rev}$. $\mathrm{min}^{-1}$, samples were removed at intervals during the ensuing growth period for measurements of cell numbers $(O)$, oxygen uptake rates $(\nabla), A_{5 s 0}(\nabla)$ and modal cell volume (O).

Fig. 3. Effects of inhibitors on respiration during the cell cycle of B. subtilis: (a) Nbf-Cl $(1.66 \mu \mathrm{M}) ;(b)$ DCCD $(166 \mu \mathrm{M}) ;(c)$ HQNO $(28 \mathrm{nM}, 0 ; 56 \mathrm{nM}, 0)$. The rate of oxygen uptake in the presence of inhibitor is expressed as a percentage of that in the absence of inhibitor. The vertical lines in each experiment refer to the mid-points of the first and second increases in cell numbers.

cells by $40 \%$ ) to samples withdrawn from a synchronous culture resulted in variable inhibition of respiration rates during the cell cycle (Fig. $3 a$ ). The halving of the inhibition at approximately 140 and $170 \mathrm{~min}$ is probably due to a doubling in cell numbers and hence inhibition sites. Maxima of inhibition occurred at 0.57 of a cycle and at cell division $(0.0)$. A similar pattern of inhibition of respiration rates occurred after the addition of $166 \mu \mathrm{M}$-DCCD (sufficient to inhibit the respiration of asynchronously dividing cells by $50 \%$ ) at different 


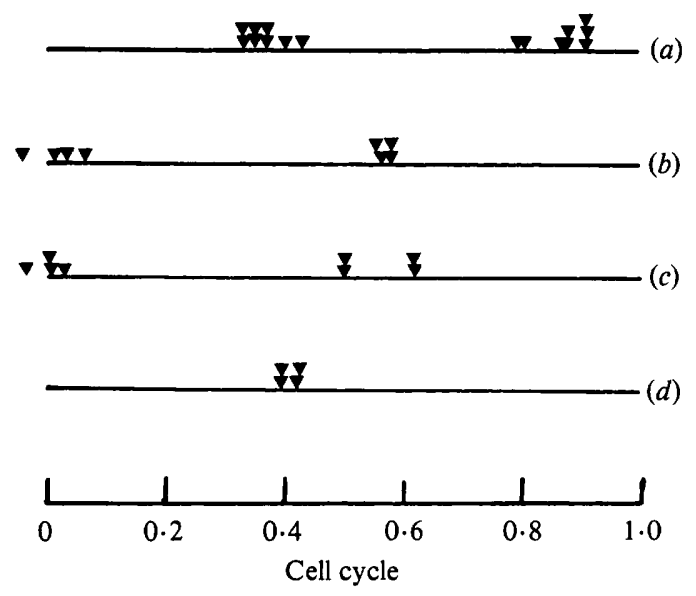

Fig. 4. Cell cycle maps summarizing the respiratory periodicities which occur during the cell cycle of $B$. subtilis. The cell cycle is shown as a linear scale, $0-1 \cdot 0$. (a) Mid-points of rises in oxygen uptake rates; (b) (c) and (d) points of maximum inhibition by $\mathrm{Nbf}-\mathrm{Cl}, \mathrm{DCCD}$ and HQNO, respectively. Map (a) shows the pooled data from eight separate experiments whilst $(b),(c)$ and $(d)$ each contain data from two separate experiments.

stages of synchronous growth (Fig. $3 b$ ). Again, inhibition maxima were apparent at 0.6 of a cycle and at cell division. $\mathrm{Nbf}-\mathrm{Cl}$ at $1.66 \mu \mathrm{M}$ had no effect on $\mathrm{NADH}$ oxidation but inhibited ATP hydrolysis in membrane preparations by approximately $93 \%$ (results not shown). DCCD at $166 \mu \mathrm{M}$ inhibited ATP hydrolysis completely in membrane preparations but resulted in a slight inhibition of NADH oxidation (14\%). It would seem, therefore, that the major effects of these inhibitors is at the energy-transfer reactions of the proton-translocating ATPase. Moreover, the use of DCCD and $\mathrm{Nbf}-\mathrm{Cl}$ during synchronous growth revealed a new pair of respiration-associated phases in the cell cycle.

The addition of HQNO to samples removed at different stages of synchronous growth also revealed discontinuities of inhibition of respiration rates (Fig. 3c). A maximum of inhibition occurred at 0.4 of a cycle, coincident with the timing of the first mid-point of the step increase in respiration. A doubling in the concentration of HQNO did not alter the periodicity of inhibition but caused a twofold increase in the extent of inhibition (Fig. $3 \mathrm{c}$ ).

\section{DISCUSSION}

Continuous-flow centrifugation is a convenient and rapid method for the preparation of synchronous cultures of $B$. subtilis. It is apparent that for the best results no more than $10 \%$ of a population can be selected (Fig. 1). For $B$. subtilis this may reflect a relatively large proportion of the cell cycle spent in cell separation. This is borne out by a division period in synchronous cultures of $40 \mathrm{~min}$ which is followed by only a short delay prior to the second division. Brown (1977) has shown that autolysins, which may be involved in cell separation, are subject to catabolite repression in $B$. subtilis grown with glucose as a carbon source. This may account for the decay in the degree of synchrony which occurs in the second cycle and at size selections greater than $10 \%$. The second division appears to be much less synchronous than the first. Determinations of modal cell volumes, however, suggest that the second division still retains a reasonable degree of synchrony. A similar decay of synchrony for glucose-grown synchronous cultures of $B$. subtilis prepared by selection using Percoll has recently been noted by Dwek et al. (1980).

The timings of the mid-points of rises in respiration rates during the cell cycle together with the points of maximum inhibition by $\mathrm{Nbf}-\mathrm{Cl}, \mathrm{DCCD}$ and HQNO from a number of experiments are summarized in Fig. 4. It has been proposed by Poole (1977) that the pattern 
of periodic increases of respiration rates in $E$. coli is governed by whether the carbon source is fermentative (rises as maxima/minima) or non-fermentative (stepwise rises). Clearly this does not hold for the results presented here for $B$. subtilis. However, the timings of step increases in respiration at 0.37 and 0.87 of a cycle are very similar to those observed for $A$. eutrophus (Edwards \& Jones, 1977) and E. coli (Evans, 1975).

The maximum inhibition by HQNO corresponds to the first step increase of respiration rate and could be due to modulation of the respiratory chain at that time. This might occur via changes in the activity of dehydrogenases or reactive centres at sites 1 or 2 . The second rise in respiration rate does not correspond to an increasing inhibition by HQNO; this may be due to changes at site 2 only, which result in a loss or masking of inhibitor-binding sites. It is probable that $\mathrm{Nbf}-\mathrm{Cl}$ and DCCD exert their effect primarily on energy-transfer reactions since they inhibit ATPase activity more than respiratory activity of membrane preparations at the concentrations used in the synchronously growing cultures. Thus, two pairs of temporal phases seem to occur in the cell cycle of glucose-grown $B$. subtilis. One pair, at 0.37 and 0.87 , is concerned with respiration whilst the other, at 0.6 and at cell division, may be concerned with energy-conserving reactions. Relatively little is known of the effects of inhibitors on respiratory events during the bacterial cell cycle. Edwards et al. (1978) have investigated the effects of DCCD and $\mathrm{Nbf}-\mathrm{Cl}$ on the ATPase activity in cell extracts of $A$. eutrophus prepared at different stages of synchronous growth. They noted that DCCD inhibited ATPase activity maximally at 0.1 and 0.6 of a cycle, intermediate to the mid-points of rises of respiration in that organism and at similar stages to those observed here for whole cells of $B$. subtilis. However, in contrast, $\mathrm{Nbf}-\mathrm{Cl}$ inhibited ATPase activity of $A$. eutrophus at the same stages as the rises in respiration. Further studies on the activities of ATPase, in the presence and absence of inhibitors, during the cell cycle of $B$. subtilis are needed to resolve these observations.

Some major cellular events have been reported to occur during the cell cycle of glucose-grown $B$. subtilis. Miyakawa et al. (1980) have shown that two transition points for protein synthesis occur during the cell cycle. One of these, at 0.64 of a cycle, is proposed as the transition point for the synthesis of division proteins and occurs at the time of septation. A doubling in the rate of membrane protein synthesis and nuclear segregation at 0.9 of a cycle was noted by Sargent (1975). Nanninga et al. (1979) studied the cell cycle of $B$. subtilis by electron microscopy and suggested that initiation of cell division, cell separation and termination of DNA replication occurred at $0.34,0.66$ and 0.92 , respectively. These reports indicate that important periodic events occur during the cell cycle of $B$. subtilis which may reflect the respiratory periodicities presented here. A change in the cell's energy requirements during the cycle is indicated. The exact causal factors remain to be elucidated but probably include periodic synthesis of macromolecules, especially proteins.

This work was supported by an S.R.C. award, GR/A58890. We are grateful to the Royal Society for a grant to purchase the Coulter counter.

\section{REFERENCES}

Beechey, R. B., Robertson, A. M., Holloway, C. T. \& KNIGHT, I. G. (1967). The properties of dicyclohexylcarbodiimide as an inhibitor of oxidative phosphorylation. Biochemistry 6, 38673872.

Blumenthal, L. K. \& Zahler, S. A. (1962). Index for measurement of synchronization of cell populations. Science 135, 724.

BRown, W. C. (1977). Autolysins in Bacillus subtilis. In Microbiology-1977, pp. 75-84. Edited by D. Schlessinger. Washington, D.C.: American Society for Microbiology.
Dwek, R. D., Kobrin, L. H., Grossman, N. \& RoN, E. Z. (1980). Synchronization of cell division in micro-organisms by Percoll gradients. Journal of Bacteriology 144, 17-21.

EDWARDS, C. (1980). Sensitivity of synchronous cultures of Bacillus subtilis to lysozyme. Journal of General Microbiology 119, 277-279.

EDWARDS, C. \& JONES, C. W. (1977). Respiratory properties of synchronous cultures of Alcaligenes eutrophus $\mathrm{H} 16$ prepared by a continuous-flow size selection method. Journal of General Microbiology 99, 383-388. 
Edwards, C., Spode, J. A. \& Jones, C. W. (1978). The properties of adenosine triphosphatase from exponential and synchronous cultures of Alcaligenes eutrophus H16. Biochemical Journal 172, 253260.

Evans, J. B. (1975). Preparation of synchronous cultures of Escherichia coli by continuous-flow size selection. Journal of General Microbiology 91, 188-190.

Ferguson, S. J., Lloyd, W. J. \& Radda, G. K. (1974). An unusual and reversible modification of soluble beef heart mitochondrial ATPase. FEBS Letters 38, 234-236.

Lloyd, D. (1974). The Mitochondria of Microorganisms. London \& New York: Academic Press.

Lloyd, D., John, L., Edwards, C. \& Chagla, A. H. (1975). Synchronous cultures of micro-organisms: large-scale preparation by continuous-flow size selection. Journal of General Microbiology 88, 153-158.

Miyakawa, Y., Komano, T. \& Maruyama, Y. (1980). Cell-cycle inhibition by chloramphenicol of septum formation and cell division in synchronized cells of Bacillus subtilis. Journal of Bacteriology 141, 502-507.

NANninga, N., Koppes, L. J. H. \& De VRies-Tiussen, F. C. (1979). The cell cycle of Bacillus subtilis as studied by electron microscopy. Archives of Microbiology 123, 173-181.

Poole, R. K. (1977). The influence of growth substrate and capacity for oxidative phosphorylation on synchronous cultures of Escherichia coli K12. Journal of General Microbiology 99, 369-377.

Poole, R. K. \& Lloyd, D. (1974). Changes in respiratory activities during the cell cycle of the fission yeast Schizosaccharomyces pombe $972 \mathrm{~h}^{-}$ growing in the presence of glycerol. Biochemical Journal 144, 141-148.

SARGENT, M. G. (1975). Control of membrane synthesis in Bacillus subtilis. Biochimica et biophysica acta 406, 564-574.

SlATER, E. C. (1967). Application of inhibitors and uncouplers for a study of oxidative phosphorylation. Methods in Enzymology 10, 48-57. 